\title{
Empirical estimation of sequencing error rates using smoothing splines
}

\author{
Xuan Zhu' ${ }^{1}$, Jian Wang ${ }^{1}$, Bo Peng ${ }^{2}$ and Sanjay Shete ${ }^{1,3^{*}}$
}

\begin{abstract}
Background: Next-generation sequencing has been used by investigators to address a diverse range of biological problems through, for example, polymorphism and mutation discovery and microRNA profiling. However, compared to conventional sequencing, the error rates for next-generation sequencing are often higher, which impacts the downstream genomic analysis. Recently, Wang et al. (BMC Bioinformatics 13:185, 2012) proposed a shadow regression approach to estimate the error rates for next-generation sequencing data based on the assumption of a linear relationship between the number of reads sequenced and the number of reads containing errors (denoted as shadows). However, this linear read-shadow relationship may not be appropriate for all types of sequence data. Therefore, it is necessary to estimate the error rates in a more reliable way without assuming linearity. We proposed an empirical error rate estimation approach that employs cubic and robust smoothing splines to model the relationship between the number of reads sequenced and the number of shadows.

Results: We performed simulation studies using a frequency-based approach to generate the read and shadow counts directly, which can mimic the real sequence counts data structure. Using simulation, we investigated the performance of the proposed approach and compared it to that of shadow linear regression. The proposed approach provided more accurate error rate estimations than the shadow linear regression approach for all the scenarios tested. We also applied the proposed approach to assess the error rates for the sequence data from the MicroArray Quality Control project, a mutation screening study, the Encyclopedia of DNA Elements project, and bacteriophage PhiX DNA samples.

Conclusions: The proposed empirical error rate estimation approach does not assume a linear relationship between the error-free read and shadow counts and provides more accurate estimations of error rates for next-generation, short-read sequencing data.
\end{abstract}

Keywords: Empirical error rate, Next-generation sequencing, Smoothing spline, Frequency-based simulation, Short reads

\section{Background}

Next-generation sequencing usually refers to massively parallel high-throughput DNA sequencing technologies that can sequence millions of small DNA fragments at the same time $[1,2]$. Next-generation sequencing has developed rapidly and is used in a diverse range of biological investigations, such as quantification of gene expression, polymorphism and mutation discovery, microRNA profiling, and genome-wide mapping of protein-DNA interaction

\footnotetext{
* Correspondence: sshete@mdanderson.org

'Department of Biostatistics, The University of Texas MD Anderson Cancer Center, Houston, TX 77030, USA

${ }^{3}$ Department of Epidemiology, The University of Texas MD Anderson Cancer Center, Houston, TX 77030, USA

Full list of author information is available at the end of the article
}

[3-8]. Next-generation sequencing costs much less than conventional Sanger sequencing techniques because its high-throughput capacity enables a much higher degree of parallelism and much smaller reaction volumes $[7,9]$. On the other hand, next-generation sequencing produces large numbers of short-read-length sequences $[10,11]$, which are difficult to correctly and fully assemble [12]. In addition, the error rates for next-generation sequencing are often higher than those for conventional Sanger sequencing, which negatively impacts downstream genomic analyses that use next-generation sequencing data $[7,9,13,14]$. Therefore, before genomic analyses can be performed, quality assessment of the next-generation sequencing reads is essential [15]. These assessments include measuring intrinsic quality metrics (FastQC) [16], sequence coverage, 
sequence error rates, and paired-end, fragment-size distributions $[15,17]$.

Different approaches to estimating sequence error rates have been proposed, with or without the use of a reference genome. Bullard et al. [18] aligned the sequencing reads to a reference genome to obtain the numbers of uniquely mapped reads with 0,1 , or 2 mismatches, and the per-read sequencing error rate was calculated as the proportion of reads with at least one error. This mismatch counting approach assumed that reads with 0 mismatch had no errors and reads with 1 or 2 mismatches were errors but not polyphorphisms [7]. Also, this approach depends on a well-established reference, which might not be available when sequencing a new genome [17]. The error correction tools based on $k$-mer $[15,19-25]$ can also provide estimations for sequencing error rates. These approaches are reference free and less sensitive to errors that occur due to polymorphisms [26]. However, since the $k$-mer-based approaches usually involve computing the frequencies of all distinct substrings of length $k$ appearing in the sequence, these approaches are computationally demanding and require a large amount of computer memory, making it difficult for them to process reads from large genomes [27-29].

Recently, Wang et al. [7] developed a novel approach called shadow regression to estimate short sequencing read error rates without a reference genome. These authors observed that the number of shadows due to sequencing errors increases linearly with the number of reads sequenced. The per-read error rate was defined as the proportion of reads containing sequencing errors among all the reads in a sample. A linear regression model was employed to assess the error rates (details will be reviewed in the Method section). However, in practice, the linear assumption for the relationship between the number of reads sequenced and the number of reads that contain errors might not be appropriate for all types of sequence data (see the sample data from [7], Additional file 1). In these situations, the shadow regression based on a linear assumption might lead to a biased estimation of the sequencing error rates.

Therefore, in this study, we proposed an empirical approach to estimate the sequencing error rates that uses cubic smoothing spline and robust smoothing spline methods to model the relationship between the number of reads sequenced and the number of reads that contain errors. We took the per-read error rate used by Wang et al. [7] and redefined it as a function of the read counts. We also defined a sample per-read error rate as the median of the per-read error rates obtained using different numbers of read counts and corresponding fitted counts of reads containing errors. To better investigate the performance of the proposed approach, we developed a frequency-based simulation approach based on the sequencing reads from real data sets that mimics the nonlinear relationship between the numbers of reads sequenced and the numbers of reads that contain errors more realistically than the Wang et al.'s simulation approch. We performed the simulation studies using sequence sample data from the MicroArray Quality Control (MAQC) project [30], a mutation screening study [31], the Encyclopedia of DNA Elements (ENCODE) project [32], and bacteriophage PhiX 174RF1 DNA samples [7] and compared the results with those obtained by using shadow linear regression. The proposed empirical approach provided more accurate error rate estimations than shadow linear regression for all the scenarios in which the assumption of linearity was not valid and provided similar error rates when the assumption of linearity was valid. We also applied the empirical error rate estimation approach to assess the sequencing error rates for real sample data from MAQC, mutation screening, ENCODE, and PhiX DNA samples.

\section{Methods \\ Shadow regression overview}

Wang et al. [7] made the important observation that the number of shadows due to sequencing errors increases linearly with the number of reads sequenced, whereas the number of true shadows is independent of the number of reads sequenced. In the current study, we employed and modified the definitions and notations from the Wang et al. [7] study.

Specifically, given a sequence $t$ in a sample, the total number of reads can be given as $r_{t}=n_{t}+e_{t}$, where, $r_{t}$ is the total number of reads with sequence $t, n_{t}$ is the number of reads that are error free with sequence $t$, and $e_{t}$ is the number of reads that contain sequencing errors with sequence $t$. In practice, one would not know the true error-free sequence reads. Wang et al. first converted a sequence file (i.e., FASTQ format with equal length for all reads) from a sample into a read counts file. The number of reads for each sequence was counted over the sample, and then the authors ranked all the sequences according to the read counts (see an example of read counts in Additional file 2). The top 1000 reads with the highest frequencies in a sample were selected as the error-free sequences and used to calculate $n_{t}$ and $e_{t}$ in a different sequence $t$. The shadows of a given sequence $t$ were defined as the reads differing from the error-free reads by up to two bases, which was deemed by Wang et al. [7] to be sufficiently similar to the given error-free sequence $t$ to estimate substitution errors. Finally, the top 1000 reads with the highest frequencies in a sample were excluded from the assessment of the shadow counts.

The per-read error rate was defined as the proportion of reads containing sequencing errors over all the reads 
in a given sample [7], or $E R=\frac{\sum_{t} e_{t}}{\sum_{t} r_{t}}=\frac{\Delta e_{t}}{\Delta r_{t}}$. Based on the observation that the number of shadows due to the sequencing errors increases linearly with the number of reads, Wang et al. proposed a linear model as $s_{t}=\alpha+\beta n_{t}$ $+\epsilon$, where $s_{t}$ is the number of shadows of sequence $t$ and $\epsilon$ is the independent error that follows approximately Gaussian distribution. Robust linear regression was used to estimate the coefficients $\alpha$ and $\beta$ because shadows can come from legitimate error-free reads. In this situation, the per-read error rate can be estimated by the slope of the linear model as $E R=\frac{\Delta e_{t}}{\Delta r_{t}}=\frac{\Delta e_{t}}{\Delta n_{t}+\Delta e_{t}}=\frac{\beta}{1+\beta}$, which was denoted as the shadow regression error rate (SRER) in the current study.

\section{Empirical per-read error rate with smoothing splines}

The error rates assessed by shadow linear regression were based on the assumption of a linear relationship between the error-free read counts and shadow counts. However, the real data examples in Wang et al.'s paper (e.g., Additional file 1) show that for many types of sequencing data the relationship between error-free read counts and shadow counts does not follow a linear trend. In these situations, shadow regression based on a linear assumption might lead to a biased estimation of the sequence error rate. Therefore, in this study, we employed the cubic smoothing spline and robust smoothing spline methods to model the relationship between the error-free read counts and the shadow counts; the piecewise curve resulting from these spline methods is capable of capturing relationships of widely varying form and tends to avoid erratic behavior near the extremes of the data [33]. Based on the empirical read-shadow relationship, we proposed the error rate estimation as a function of error-free read counts, which we hypothesized would be more useful in practice than the shadow regression approach for estimating error rates.

We first used the cubic smoothing spline method [34, 35 ] to model the shadow counts $\left(s_{t}\right)$ as a function of the error-free read counts $\left(n_{t}\right)$. The cubic smoothing spline method fits a smooth curve to a set of observations using a cubic function [35, 36]. Specifically, given a set of observations of error-free read counts and shadow counts, $\left(n_{1}, s_{1}\right),\left(n_{2}, s_{2}\right), \ldots,\left(n_{m}, s_{m}\right), n_{1}<n_{2}<\ldots<n_{m}$, we modeled the relationship between $n_{i}$ and $s_{i}$ as a function $s_{i}=\mathcal{U}\left(n_{i}\right)$, with two continuous derivatives, where $n_{i}$ is the number of error-free read counts with sequence $i, s_{i}$ is the number of shadow counts with sequence $i, i=1$, ..., $m$, and $m$ is the total number of sequences. Among all such functions with two continuous derivatives, the purpose of the smoothing spline is to find the estimated function minimizing the penalized residual sum of squares $\Sigma_{1}^{m}\left(s_{i}-\hat{U}\left(n_{i}\right)\right)^{2}+\lambda \int_{n_{1}}^{n_{m}} \hat{U}^{\prime \prime}(n)^{2} d n$, where $\lambda$ is a smoothing parameter [35]. We used the cubic smoothing spline implemented in the $\mathrm{R}$ function "smooth.spline" in the "stats" package. To improve the robustness of the spline, we used a robust smoothing spline approach to model the relationship between shadow counts $\left(s_{t}\right)$ and error-free read counts $\left(n_{t}\right)$. This approach uses an iterative re-weighted smoothing spline algorithm with the inverse of the absolute value of the residuals as the weights. We used the robust smoothing spline implemented in the $\mathrm{R}$ function "robustSmoothspline" in the package "aroma.light" [37, 38].

Based on the fitted model of shadow counts as a spline function of error-free read counts, we used the definition of the per-read error rate used by Wang et al. [7]. Let $n_{1}$, $n_{2}, \ldots, n_{m}$ be the read counts of $m$ sequences, where $n_{1}<n_{2}, \ldots, n_{m-1}<n_{m}$, and let $\hat{S}_{1}, \hat{S}_{2}, \ldots, \hat{S}_{\mathrm{m}}$ be the corresponding fitted values of shadow counts using the smoothing spline approaches described above. Given a sequence $t$ with read count $n_{t}$ and fitted shadow count $\hat{S}_{\mathrm{t}}$, we defined the per-read error rate as

$$
E R\left(n_{t}\right)=\frac{\sum_{j=1}^{t} \hat{S}_{j}}{\sum_{j=1}^{t} \hat{S}_{j}+\sum_{j=1}^{t} n_{j}} .
$$

Estimation of error rates using this definition also assumes the increasing number of shadows with the increasing number of read counts in the presence of sequencing errors, but this definition allows error rates to vary by read counts, whereas the Wang et al. [7] approach assumes the error rate to be a constant irrespective of the number of reads. For the purpose of comparison with the Wang et al. [7] approach, we also defined a sample per-read empirical error rate. Given a sample with $m$ sequences, we randomly sampled 1000 numbers of read counts from $n_{1}$ to $n_{m}$ and calculated the per-read error rates for each of these read counts using the fitted spline. The median of these per-read error rates was reported as a sample per-read empirical error rate (henceforth denoted as EER).

\section{Next-generation sequencing data}

We used next-generation sequencing data from four projects-the MicroArray Quality Control (MAQC) project (mRNA-seq) [30], a mutation screening study (re-sequencing) [31], the Encyclopedia of DNA Elements (ENCODE) project (mRNA-seq) [32], and bacteriophage PhiX 174RF1 (PhiX) DNA samples [7]-to perform the simulations and demonstrate the accuracy of the proposed empirical error rate estimation approach. PhiX DNA data in FASTQ format were generated by the Center for Cancer 
Computational Biology at Dana-Farber Cancer Institute and provided by Wang et al. [7]. The other three data sets are publicly available from the National Center for Biotechnology Information Sequence Read Archive [39] in FASTQ format with equal read lengths in each sample. All the data were converted to read counts using the shadow regression program provided by Wang et al. [7].

\section{MAQC brain experiment 2 data}

The MAQC project was initiated to address concerns about the reliability of microarray technology [30]. This project provided gene expression levels measured from two RNA samples, including a Universal Human Reference RNA from Stratagene and a Human Brain Reference RNA from Ambion, in four titration pools on seven microarray platforms with three expression methodologies. In this study, we used the sequence data from the MAQC brain experiment 2 (Sequence Read Archive [SRA]010153), including 14 samples on two flowcells (SRX016366 and SRX016368) run on the Illumina 1G Genome Analyzer with each sample containing 12 million reads.

\section{Mutation screening resequencing data}

The mutation screening study provided re-sequencing data (SRA010105) from 24 patients with X-linked mental retardation (XLMR) for mutations in 86 previously identified XLMR genes, using a method that combined a novel droplet-based multiplex PCR method and nextgeneration sequencing [31]. An Illumina/Solexa Genome Analzer II platform was used to perform the sequencing, and each sample contained $\sim 12$ million reads.

\section{ENCODE transcriptome data}

The ENCODE project used high-throughput approaches to provide a biologically more informative representation of the human genome [32]. The ENCODE pilot phase included more than 200 experimental and computational data sets from 35 groups [32]. In this study, we used the ENCODE human mRNA sequence data (SRA001150), including 5 samples of human cell line K562 (SRX000570) run on the Illumina 1G Genome Analyzer; each sample contained $\sim 12$ million reads.

\section{PhiX DNA data}

The bacteriophage PhiX 174 is an icosahedral virus. It contains a closed circular single-stranded DNA molecule with 5386 nucleotide bases [40]. PhiX 174 was the first DNA-based genome for which the complete nucleotide sequence was successfully determined [40-42]. In this study, we used two PhiX DNA samples provided by Wang et al., which were generated from the Center for Cancer Computational Biology at Dana-Farber [7]. The
PhiX DNA samples were sequenced using Illumina. One sample contained $\sim 14.6$ million reads, and the other contained $\sim 25.7$ million reads.

\section{Simulation approaches}

We performed simulation studies to investigate the performance of the proposed EER and compare it with the SRER. We used two approaches to perform the simulation: (1) Wang et al.'s simulation approach and (2) a new frequency-based simulation approach described below.

\section{Wang et al. simulation approach}

In the Wang et al. study [7], the simulation was conducted based on a sample from the MAQC brain experiment 2 (SRR037440) using calibration. The authors considered reads uniquely mapped to the reference genome with no mismatches as error-free reads and then added substitution errors based on pre-specified base-specific error rates, which were estimated from the sample SRR037440 by counting the number of mismatches to the reference genome at each base. To mimic the Wang et al. simulation procedure, we used the same sample (SRR037440) to retrieve the error-free reads and estimate the pre-specified base-specific error rates. In particular, we retrieved $\sim 4.4$ million perfectly matched reads from the SRR037440 sample, which originally had $\sim 12$ million reads. We used several approaches to estimate the pre-specified base-specific error rates. In our first approach, we aligned the reads to the reference genome, marked the mismatch locations, and then calculated base-specific error rates as a percentage of the mismatch nucleotides at each location (Additional file 3(C)). In our second approach, we located all unprocessed reads for each sequence, recorded locations and numbers of mismatch nucleotides, and calculated the base-specific error rates from the total number of mismatch nucleotides at each location (Additional file 3(D)). However, we found that neither of these approaches could obtain the same base-specific error rates shown in the Wang et al. study. Thus, we included the pre-specified base-specific error rates used in their study, which we extracted approximately from the figures in the article [7] (Additional file 3(E)). In addition, we used the basespecific error rates for sample SRR037440 obtained from the shadow regression software developed by Wang et al. [7] as another set of pre-specified base-specific error rates (Additional file 3(F)).

The Wang et al. simulation approach assumed an underlying linear relationship between the error-free read counts and shadow counts, no matter which basespecific error rate was used. Therefore, the data simulated by using the Wang et al. simulation approach would not reflect the non-linear relationships between error-free read counts and shadow counts (Additional file 3(A)). For example, in some samples, when the number of error-free 
read counts is low, the shadow counts may decrease as the error-free read counts increase. Therefore, we proposed a frequency-based simulation approach to generate the error-free read counts and shadow counts directly, which can mimic the real sequence counts of the data structure.

\section{Frequency-based simulation approach}

Given the counts of all sequence reads in a real sample, we followed the same procedure described in Wang et al. to obtain the error-free read counts $\left(n_{i}\right)$ and shadow counts $\left(s_{i}\right)$ for $N$ sequence reads, $i=1,2, \ldots N$ and $n_{1}<n_{2}, \ldots, n_{N-1}<n_{N}$. We selected the top $N$ reads with the highest frequencies to be the error-free reads and obtained the corresponding read counts $\left(n_{i}\right)$; we then mapped all the rest of the sequences in the sample to the error-free reads to identify the corresponding shadow counts $\left(s_{i}\right)$. We used $N=1000$, as suggested in Wang et al. Based on the read counts and shadow counts in the real sample, we first constructed a frequency table for the error-free read counts $\left(n_{i}\right)$ using pre-specified bin widths. Because in most of the samples we investigated, the error-free read counts became very sparse when they were large, we used unequal bin widths to avoid having almost empty bins. Within each bin of the error-free read counts, we then constructed a frequency table for the shadow counts $\left(s_{i}\right)$ using prespecified equal bin widths. Given $M$ bins for error-free read counts and $L$ bins for shadow counts within each of the error-free read count bins, the frequencies can be written as $p_{j}, j=1,2, \ldots, M$, for error-free read counts and $q_{k j}, k=1,2, \ldots, L$ and $j=1,2, \ldots, M$, for shadow counts. To sample a pair of observations $\left(n_{\text {new }}, s_{\text {new }}\right)$, we first sampled two independent random numbers, $U \sim$ uniform $(0,1)$ and $V \sim$ uniform $(0,1)$. If $U \in\left[\sum_{i=1}^{j} p_{i}, \sum_{i=1}^{j+1} p_{i}\right)$, we sampled $n_{\text {new }} \sim \operatorname{uniform}\left(n_{j}, n_{j+1}\right)$, where $n_{j}$ and $n_{j+1}$ are the endpoints for the corresponding bin $j$ of read counts. Further, within the read counts bin $j$, if $V \in\left[\sum_{i=1}^{k} q_{i j}, \sum_{i=1}^{k+1}\right.$ $\left.q_{i j}\right)$, we sampled $s_{\text {new }} \sim \operatorname{uniform}\left(s_{k}, s_{k+1}\right)$, where $s_{k}$ and $s_{k+1}$ are the endpoints for the corresponding bin $k$ of shadow counts. With this approach, we can generate more pairs of error-free read and shadow counts (i.e., > 1000).

A comparison between the Wang et al. simulation approach and our proposed frequency-based simulation approach, using the SRR037440 sample, showed that unlike the frequency-based approach, the Wang et al. simulation approach does not mimic the observed nonlinear data relationship between error-free read and shadow counts (Additional files 3 and 4). Therefore, we applied only the proposed frequency-based simulation approach in the simulation studies. We generated data based on the information from samples from the four data sets described above. For each sample, the median EER was calculated based on 1000 replicates and compared with the median SRER. For all the simulations, we considered the top 1000 reads of the sample of interest with the highest frequencies as the error-free reads and then determined the shadow counts accordingly, which were then used to generate the frequency tables for the simulations. Based on the frequency tables, we generated 1000 pairs of error-free reads and shadow counts for each replicate. For the simulation data sets, we also defined an expected per-read error rate as $\sum_{i} s_{i} /$ $\left(\sum_{i} s_{i}+\sum_{i} n_{i}\right)$, where $s_{i}$ and $n_{i}$ are the shadow count and error-free read count, respectively, for sequence $i, i=1$, ..., $M$. Note that $M=1000$ in the simulation studies, as we generated 1000 pairs of error-free read and shadow counts.

\section{Results}

Simulation results

As shown in Additional files 3 and 4, using the frequency-based simulation approach can better capture the relationship between error-free read and shadow counts, therefore, we used this simulation approach to perform further simulations based on next-generation sequencing data from the MAQC, mutation screening, ENCODE, and PhiX DNA sample data sets. We compared the performance of our proposed EER approach using the cubic or robust smoothing spline method (EER_CS or EER_RS, respectively) with that of the SRER approach.

\section{Simulation results for MAQC data}

Table 1 shows the median error rates (based on 1000 replicates) obtained using the shadow linear regression approach and the proposed smoothing spline approaches, based on the next-generation sequencing data from the MAQC study. We have also reported the expected error rates (calculated as described above) and the estimation biases, which were calculated as the absolute differences between the estimated error rates and the expected error rates. For all 14 samples of MAQC data, both smoothing spline approaches provided more accurate estimations of the error rates with less bias than the shadow linear regression approach. Both smoothing spline approaches performed very similarly. For example, for sample SRR 037452, the median expected error rate in the simulation was 0.3305 . Using SRER, the median estimated error rate was 0.2578 , with a bias of 0.0727 compared to the expected error rate. In contrast, EER_CS and EER_RS had median estimated error rates of 0.3104 and 0.3096 , respectively, with biases of 0.0201 and 0.0209 , respectively. From these results, we can observe that the SRER approach underestimated the error rates, while the smoothing spline approaches provided more accurate estimated error rates. 
Table 1 Median error rates in MAQC data using shadow linear regression and smoothing spline approaches ${ }^{2}$

\begin{tabular}{|c|c|c|c|c|c|c|c|}
\hline Samples & Expected ER & SRER & SRER Bias & EER_CS & EER_CS Bias & EER_RS & EER_RS Bias \\
\hline SRR037452 & 0.3305 & 0.2578 & 0.0727 & 0.3104 & 0.0201 & 0.3096 & 0.0209 \\
\hline SRR037453 & 0.1917 & 0.1584 & 0.0333 & 0.1824 & 0.0093 & 0.1818 & 0.0099 \\
\hline SRR037454 & 0.2354 & 0.1515 & 0.0839 & 0.2060 & 0.0294 & 0.2059 & 0.0295 \\
\hline SRR037455 & 0.1759 & 0.1448 & 0.0311 & 0.1675 & 0.0084 & 0.1668 & 0.0091 \\
\hline SRR037456 & 0.2312 & 0.1622 & 0.0690 & 0.2037 & 0.0275 & 0.2035 & 0.0277 \\
\hline SRR037457 & 0.1841 & 0.1480 & 0.0361 & 0.1777 & 0.0064 & 0.1771 & 0.0070 \\
\hline SRR037458 & 0.2653 & 0.2321 & 0.0332 & 0.2582 & 0.0071 & 0.2575 & 0.0078 \\
\hline SRR037459 & 0.2371 & 0.1943 & 0.0428 & 0.2202 & 0.0169 & 0.2203 & 0.0168 \\
\hline SRR037460 & 0.2530 & 0.2018 & 0.0512 & 0.2503 & 0.0027 & 0.2490 & 0.0040 \\
\hline SRR037461 & 0.2180 & 0.1704 & 0.0476 & 0.2105 & 0.0075 & 0.2104 & 0.0076 \\
\hline SRR037462 & 0.2443 & 0.1734 & 0.0709 & 0.2322 & 0.0121 & 0.2308 & 0.0135 \\
\hline SRR037463 & 0.2154 & 0.1654 & 0.0500 & 0.2023 & 0.0131 & 0.2045 & 0.0109 \\
\hline SRR037464 & 0.2624 & 0.1666 & 0.0958 & 0.2392 & 0.0232 & 0.2403 & 0.0221 \\
\hline SRR037465 & 0.2145 & 0.1742 & 0.0403 & 0.2038 & 0.0107 & 0.2037 & 0.0108 \\
\hline
\end{tabular}

${ }^{\mathrm{a}}$ Based on 1000 replicates. The frequency-based simulation approach was applied. For each replicate, we considered the top 1000 reads with the highest frequencies as the error-free reads and generated 1000 pairs of error-free read counts and shadow counts

Expected $E R$ expected error rate in simulation studies

SRER error rate estimated using shadow regression

SRER Bias the absolute value of the difference between SRER and Expected ER

$E E R \_C S$ empirical error rate estimated using cubic smoothing spline

$E E R \_C S$ Bias the absolute value of the difference between EER_CS and Expected ER

$E E R \_R S$ empirical error rate estimated using robust smoothing spline

$E E R \_R S$ Bias the absolute value of the difference between EER_RS and Expected ER

\section{Simulation results for mutation screening resequencing data}

Table 2 reports the median error rates obtained using the SRER approach and the two EER approaches, based on next-generation sequencing data from a mutation screening study. Similar to the results from the MAQC samples, all 24 mutation screening resequencing samples yielded error rates for the smoothing spline approaches that were more accurate than or comparable to the estimations of error rates for SRER. For example, in sample SRR032566, the median of the expected error rate in the simulation was 0.0734. Using SRER, the median of the estimated error rate was 0.0412 , with a bias of 0.0323 compared with the expected error rate. Using the smoothing spline approaches, the median of the estimated error rate was 0.0705 for both approaches, with a very small bias of 0.0029 .

\section{Simulation results for ENCODE transcriptome data}

Table 3 reports the median error rates obtained using different approaches based on next-generation sequencing data from the ENCODE study. The five samples from the ENCODE study had higher expected error rates than the samples in the MAQC and mutation screening studies, which might have been due to relatively large shadow counts that corresponded with smaller error-free read counts Additional file 1(C). In this situation, the smoothing spline approaches still performed better than shadow linear regression. For example, in sample SRR002056, the expected error rate was 0.3646, and the estimated error rates were 0.2906, 0.3270, and 0.3233 for SRER, EER_CS, and EER_RS, respectively, with biases of $0.0740,0.0376$, and 0.0413 , respectively.

\section{Simulation results for PhiX DNA data}

Table 4 reports the median error rates obtained using different approaches based on the next-generation sequencing data from two PhiX DNA samples. For both samples, the smoothing spline approaches provided more accurate estimations of the error rates than did shadow linear regression. For example, in sample 100217, the median of the expected error rate in the simulation was 0.0323. Using SRER, the median of the estimated error rate was 0.0250 , with a bias of 0.0073 compared to the expected error rate. The median estimated error rate for EER_CS and EER_RS was 0.0315, resulting in a much smaller bias of 0.0008 for both approaches.

\section{Next-generation sequencing data analysis results}

We applied our smoothing spline approaches to evaluate the error rates for the next-generation sequencing data from MAQC, mutation screening, ENCODE and PhiX DNA samples, and compared error rates using our smoothing spline approaches and shadow linear regression. The estimated error rates are reported in Tables 5, 6, 7 and 8, respectively, for samples from the MAQC, 
Table 2 Median error rates in mutation screening data using shadow linear regression and smoothing spline approaches ${ }^{a}$

\begin{tabular}{|c|c|c|c|c|c|c|c|}
\hline Samples & Expected ER & SRER & SRER Bias & EER_CS & EER_CS Bias & EER_RS & EER_RS Bias \\
\hline SRR032565 & 0.1991 & 0.0493 & 0.1498 & 0.1080 & 0.0911 & 0.1082 & 0.0910 \\
\hline SRR032566 & 0.0734 & 0.0412 & 0.0323 & 0.0705 & 0.0029 & 0.0705 & 0.0029 \\
\hline SRR032567 & 0.2003 & 0.0542 & 0.1461 & 0.1111 & 0.0892 & 0.1111 & 0.0893 \\
\hline SRR032568 & 0.2040 & 0.0437 & 0.1603 & 0.1057 & 0.0984 & 0.1072 & 0.0968 \\
\hline SRR032569 & 0.1598 & 0.0509 & 0.1089 & 0.1018 & 0.0580 & 0.1014 & 0.0585 \\
\hline SRR032570 & 0.0985 & 0.0641 & 0.0345 & 0.0959 & 0.0026 & 0.0954 & 0.0032 \\
\hline SRR032571 & 0.1236 & 0.0575 & 0.0661 & 0.1406 & 0.0170 & 0.1406 & 0.0170 \\
\hline SRR032572 & 0.1495 & 0.0530 & 0.0965 & 0.1181 & 0.0314 & 0.1173 & 0.0323 \\
\hline SRR032573 & 0.1779 & 0.0912 & 0.0867 & 0.1518 & 0.0261 & 0.1506 & 0.0273 \\
\hline SRR032574 & 0.0986 & 0.0384 & 0.0602 & 0.0626 & 0.0361 & 0.0618 & 0.0368 \\
\hline SRR032575 & 0.1169 & 0.0839 & 0.0330 & 0.1228 & 0.0059 & 0.1227 & 0.0058 \\
\hline SRR032576 & 0.1554 & 0.0945 & 0.0609 & 0.1887 & 0.0333 & 0.1880 & 0.0326 \\
\hline SRR032577 & 0.1052 & 0.0694 & 0.0359 & 0.1054 & 0.0002 & 0.1055 & 0.0002 \\
\hline SRR032578 & 0.1143 & 0.0448 & 0.0695 & 0.1077 & 0.0067 & 0.1076 & 0.0067 \\
\hline SRR032580 & 0.0870 & 0.0619 & 0.0251 & 0.1169 & 0.0298 & 0.1163 & 0.0292 \\
\hline SRR032581 & 0.0770 & 0.0347 & 0.0424 & 0.0752 & 0.0018 & 0.0751 & 0.0019 \\
\hline SRR032582 & 0.0400 & 0.0041 & 0.0359 & 0.0309 & 0.0091 & 0.0310 & 0.0090 \\
\hline SRR032583 & 0.1224 & 0.0707 & 0.0517 & 0.1279 & 0.0055 & 0.1280 & 0.0056 \\
\hline SRR032584 & 0.1290 & 0.0540 & 0.0750 & 0.1052 & 0.0238 & 0.1032 & 0.0259 \\
\hline SRR032586 & 0.0445 & 0.0102 & 0.0343 & 0.0287 & 0.0158 & 0.0287 & 0.0159 \\
\hline SRR032587 & 0.1486 & 0.0786 & 0.0700 & 0.1562 & 0.0076 & 0.1552 & 0.0066 \\
\hline SRR032588 & 0.1240 & 0.0470 & 0.0770 & 0.1024 & 0.0216 & 0.1021 & 0.0220 \\
\hline SRR033543 & 0.1151 & 0.0587 & 0.0564 & 0.0828 & 0.0323 & 0.0832 & 0.0318 \\
\hline SRR033544 & 0.1267 & 0.0524 & 0.0743 & 0.1086 & 0.0181 & 0.1085 & 0.0182 \\
\hline
\end{tabular}

${ }^{a}$ Based on 1000 replicates. The frequency-based simulation approach was applied. For each replicate, we considered the top 1000 reads with the highest frequencies as the error-free reads and generated 1000 pairs of error-free read counts and shadow counts

Expected ER expected error rate in simulation studies

$S R E R$ error rate estimated using shadow regression

SRER Bias the absolute value of the difference between SRER and Expected ER

$E E R \_C S$ empirical error rate estimated using cubic smoothing spline

$E E R \_C S$ Bias the absolute value of the difference between EER_CS and Expected ER

$E E R \_R S$ empirical error rate estimated using robust smoothing spline

$E E R \_R S$ Bias the absolute value of the difference between EER_RS and Expected ER

Table 3 Median error rates in ENCODE data using shadow linear regression and smoothing spline approaches ${ }^{a}$

\begin{tabular}{lclccccc}
\hline Samples & Expected ER & SRER & SRER Bias & EER_CS & EER_CS Bias & EER_RS & EER_RS Bias \\
\hline SRR002053 & 0.5548 & 0.4153 & 0.1395 & 0.4609 & 0.0939 & 0.4565 & 0.0983 \\
SRR002056 & 0.3646 & 0.2906 & 0.0740 & 0.3270 & 0.0376 & 0.3233 & 0.0413 \\
SRR002065 & 0.4578 & 0.3371 & 0.1207 & 0.3740 & 0.0838 & 0.3701 & 0.0877 \\
SRR005092 & 0.6300 & 0.4047 & 0.2253 & 0.4797 & 0.1503 & 0.4727 & 0.1573 \\
SRR005093 & 0.4839 & 0.3928 & 0.0911 & 0.4221 & 0.0618 & 0.4173 & 0.0666 \\
\hline
\end{tabular}

${ }^{\mathrm{a}}$ Based on 1000 replicates. The frequency-based simulation approach was applied. For each replicate, we considered the top 1000 reads with the highest frequencies as the error-free reads and generated 1000 pairs of error-free read counts and shadow counts

Expected ER expected error rate in simulation studies

SRER error rate estimated using shadow regression

SRER Bias the absolute value of the difference between SRER and Expected ER

EER_CS empirical error rate estimated using cubic smoothing spline

EER_CS Bias the absolute value of the difference between EER_CS and Expected ER

$E E R \_R S$ empirical error rate estimated using robust smoothing spline

$E E R \_R S$ Bias the absolute value of the difference between EER_RS and Expected ER 
Table 4 Median error rates in PhiX DNA data using shadow linear regression and smoothing spline approaches ${ }^{\mathrm{a}}$

\begin{tabular}{lccccccc}
\hline Samples & Expected ER & SRER & SRER Bias & EER_CS & EER_CS Bias & EER_RS & EER_RS Bias \\
\hline 100217 & 0.0323 & 0.0250 & 0.0073 & 0.0315 & 0.0008 & 0.0315 & 0.0008 \\
100514 & 0.0152 & 0.0143 & 0.0009 & 0.0152 & 0.0000 & 0.0152 & 0.0000 \\
\hline
\end{tabular}

${ }^{a}$ Based on 1000 replicates. The frequency-based simulation approach was applied. For each replicate, we considered the top 1000 reads with the highest frequencies as the error-free reads and generated 1000 pairs of error-free read counts and shadow counts Expected $E R$ expected error rate in simulation studies

SRER error rate estimated using shadow regression

SRER Bias the absolute value of the difference between SRER and Expected ER

$E E R \_C S$ empirical error rate estimated using cubic smoothing spline

$E E R_{-}$CS Bias the absolute value of the difference between EER_CS and Expected ER

$E E R \_R S$ empirical error rate estimated using robust smoothing spline

$E E R \_R S$ Bias the absolute value of the difference between EER_RS and Expected ER

mutation screening, ENCODE, and PhiX DNA data sets. From the results we can observe that the smoothing spline approaches always provided relatively higher estimates of the error rates. For example, in sample SRR037454 from MAQC (Table 5), the estimated error rates were 0.1596, 0.2041, and 0.2196, respectively, for SRER, EER_CS, and EER_RS. This was expected given that shadow linear regression tended to underestimate the error rates in the simulation results.

\section{Discussion}

Due to its high-throughput capacity and low cost, nextgeneration sequencing has been widely used to address a diverse range of biological problems. However, because the higher error rates in next-generation short-read sequencing data can impact the downstream genomic analyses, it is critical to accurately assess these error rates before genomic analyses are performed. We have proposed an empirical approach that more accurately

Table 5 Error rates in real MAQC data using shadow linear regression and smoothing spline approaches

\begin{tabular}{llll}
\hline Samples & SRER & EER_CS & EER_RS \\
\hline SRR037452 & 0.2695 & 0.3124 & 0.3362 \\
SRR037453 & 0.1598 & 0.1819 & 0.1822 \\
SRR037454 & 0.1596 & 0.2041 & 0.2196 \\
SRR037455 & 0.1482 & 0.1694 & 0.1700 \\
SRR037456 & 0.1657 & 0.2062 & 0.2162 \\
SRR037457 & 0.1541 & 0.1796 & 0.1793 \\
SRR037458 & 0.2386 & 0.2573 & 0.2575 \\
SRR037459 & 0.1996 & 0.2216 & 0.2233 \\
SRR037460 & 0.2027 & 0.2504 & 0.2647 \\
SRR037461 & 0.1779 & 0.2058 & 0.2093 \\
SRR037462 & 0.1858 & 0.2329 & 0.2319 \\
SRR037463 & 0.1771 & 0.2019 & 0.2072 \\
SRR037464 & 0.1850 & 0.2377 & 0.2448 \\
SRR037465 & 0.1842 & 0.2019 & 0.2070 \\
\hline
\end{tabular}

SRER error rate estimated using shadow regression $E E R \_C S$ empirical error rate estimated using cubic smoothing spline $E E R \_R S$ empirical error rate estimated using robust smoothing spline estimates error rates for next-generation, short-read sequencing data than other available approaches.

In this paper, we first reviewed the shadow linear regression approach for short sequencing read error rate estimation proposed by Wang et al. [7]. The shadow regression approach was developed based on the assumption of a linear relationship between the number of reads sequenced and the number of shadows. The linear

Table 6 Error rates in real mutation screening data using shadow linear regression and smoothing spline approaches

\begin{tabular}{|c|c|c|c|}
\hline Samples & SRER & EER_CS & EER_RS \\
\hline SRR032565 & 0.0753 & 0.1167 & 0.1206 \\
\hline SRR032566 & 0.0584 & 0.0746 & 0.0745 \\
\hline SRR032567 & 0.0846 & 0.1420 & 0.1446 \\
\hline SRR032568 & 0.0686 & 0.1566 & 0.1566 \\
\hline SRR032569 & 0.0597 & 0.1015 & 0.1020 \\
\hline SRR032570 & 0.0691 & 0.0973 & 0.0954 \\
\hline SRR032571 & 0.0724 & 0.1400 & 0.1400 \\
\hline SRR032572 & 0.0818 & 0.1611 & 0.1593 \\
\hline SRR032573 & 0.1602 & 0.2756 & 0.2752 \\
\hline SRR032574 & 0.0557 & 0.1004 & 0.1053 \\
\hline SRR032575 & 0.0882 & 0.1191 & 0.1179 \\
\hline SRR032576 & 0.1101 & 0.1915 & 0.1899 \\
\hline SRR032577 & 0.0762 & 0.1282 & 0.1280 \\
\hline SRR032578 & 0.1365 & 0.1874 & 0.1873 \\
\hline SRR032580 & 0.0727 & 0.1262 & 0.1266 \\
\hline SRR032581 & 0.0981 & 0.0506 & 0.0511 \\
\hline SRR032582 & 0.0941 & 0.1689 & 0.1679 \\
\hline SRR032583 & 0.1141 & 0.2057 & 0.2042 \\
\hline SRR032584 & 0.0849 & 0.0963 & 0.0742 \\
\hline SRR032586 & 0.0623 & 0.3606 & 0.3621 \\
\hline SRR032587 & 0.0857 & 0.1524 & 0.1532 \\
\hline SRR032588 & 0.0701 & 0.1446 & 0.1440 \\
\hline SRR033543 & 0.0802 & 0.1084 & 0.1102 \\
\hline SRR033544 & 0.1175 & 0.1588 & 0.1586 \\
\hline
\end{tabular}

SRER error rate estimated using shadow regression

$E E R \_C S$ empirical error rate estimated using cubic smoothing spline $E E R \_R S$ empirical error rate estimated using robust smoothing spline 
Table 7 Error rates in real ENCODE data using shadow linear regression and smoothing spline approaches

\begin{tabular}{llll}
\hline Samples & SRER & EER_CS & EER_RS \\
\hline SRR002053 & 0.4134 & 0.4469 & 0.4453 \\
SRR002056 & 0.3225 & 0.3020 & 0.3355 \\
SRR002065 & 0.3842 & 0.3918 & 0.3913 \\
SRR005092 & 0.4628 & 0.4884 & 0.4776 \\
SRR005093 & 0.4090 & 0.4724 & 0.4013 \\
\hline
\end{tabular}

SRER error rate estimated using shadow regression

$E E R \_C S$ empirical error rate estimated using cubic smoothing spline

$E E R \_R S$ empirical error rate estimated using robust smoothing spline

assumption may be appropriate if one could plot the counts of true error-free reads and associated shadow counts obtained using only the reads containing errors. However, the sequencing data is noisy and such information is not identifiable. Therefore, the proposed approach to compute a sample-level error rate using the median of the error rates obtained at different values of error-free read counts and corresponding fitted shadow counts is likely to be more robust. From the sample next-generation sequencing data presented in the Wang et al. paper [7], the linear assumption for the read-shadow relationship might hold for the PhiX DNA sequencing data, but it is not valid for the mRNA sequencing data (MAQC and ENCODE) or the mutation screening resequencing data (Additional file 1). Therefore, we employed smoothing spline approaches to model the nonlinear read-shadow relationship and proposed an empirical approach to estimate the short-read sequencing error rates. The smoothing spline approaches can control the smoothness through the tuning parameter in the penalty term instead of the number and location of knots, which provides the same fit with fewer parameters, and in turn, reduces the likelihood of overfitting the data [25]. Although one could use the linear smoothing spline, in our paper we used the cubic smoothing spline because we desired a smoother interpolating function. Moreover, in addition to the cubic smoothing spline, to improve the robustness of the spline, we also used the robust smoothing spline, employing an iterative re-weighted smoothing spline algorithm with the inverse of the absolute value of the residuals as the weights. We then compared the proposed empirical error rate approach with the shadow linear regression approach using simulation studies. The

Table 8 Error rates in real PhiX DNA data using shadow linear regression and smoothing spline approaches

\begin{tabular}{llcc}
\hline Samples & SRER & EER_CS & EER_RS \\
\hline 100217 & 0.0261 & 0.0317 & 0.0315 \\
100514 & 0.0142 & 0.0155 & 0.0157 \\
\hline
\end{tabular}

SRER error rate estimated using shadow regression

$E E R \_C S$ empirical error rate estimated using cubic smoothing spline

$E E R \_R S$ empirical error rate estimated using robust smoothing spline results from the simulation studies showed that the shadow linear regression underestimated error rates while the proposed empirical approach provided more accurate estimations of the error rates. This was true even for the DNA sequencing data, where the linear read-shadow relationship might be valid.

In practice, the true sample genome sequence might not be the same as the reference genome sequence due to polymorphisms or duplications. Therefore, we performed additional simulations with two scenarios: (1) there is one polymorphism for every 1000 base pairs as was assumed in Wang et al. [7]; and (2) there are two base-pair duplications for every 1000 base pairs. It is important to note that these assumptions are specific for simulating the data and are not required for the shadowbased methods. Specifically, based on the reads of sample SRR037440, uniquely mapped to the reference genome with no mismatches, we added one polymorphism or two base-pair duplications per 1000 base pairs and considered the resulting reads as error-free reads. We then added substitution errors based on pre-specified base-specific error rates. In both scenarios, our proposed approach provided accurate results. For example, in the scenario where we assumed that there was one polymorphism per 1000 base pairs, the expected error rate in the simulation was 0.1977 . The estimated error rates using the cubic smoothing spline and robust smoothing spline were 0.1973 and 0.1989 , respectively. In the scenario where we assumed that there were two base-pair duplications per 1000 base pairs, the expected error rate in the simulation was 0.1728 . The estimated error rates were 0.1722 and 0.1761 , respectively, using the cubic smoothing spline and robust smoothing spline. These results showed that the proposed approach is not affected by the polymorphisms or duplications as well as the validity of the shadow-based approaches.

We applied the proposed approach to real data from studies of MAQC, mutation screening, ENCODE, and PhiX DNA and compared the results with those obtained using the shadow linear regression approach. The results showed that shadow regression provided relatively low error rates compared to the proposed approach. For example, for the MAQC mRNA sequencing data, our approach yielded error rates between $\sim 17$ and $\sim 32 \%$, whereas shadow regression provided error rates of $\sim 15$ to $\sim 27 \%$. The lower error rates estimated by the shadow regression method could be attributed to the linear assumption, which is not valid in some real data sets, as can be seen by even simple visual inspection (Additional file 1). When analyzing the DNA sequencing data, for which the linear relationship might be valid, the proposed approach provided estimations of error rates similar to those obtained using shadow linear regression. 
To better investigate the performance of the proposed approach, we developed a frequency-based simulation approach to capture the nonlinear relationship between the number of reads sequenced and the number of reads that contained errors based on the sequencing reads from real data sets. We compared these two simulation approaches and showed that in the data generated using the Wang et al. simulation approach the shadow counts increased linearly as the error-free read counts increased, whereas in the data generated using our frequency-based simulation approach, the patterns were similar to those in the original sample data (Additional files 3 and 4).

For the proposed empirical error rates, we adapted the original definition of per-read error rate used by Wang et al. [7], which was the proportion of reads containing sequencing errors among all the reads in a sample. Instead of using the slope from the linear model, we defined the empirical error rate as a function of the error-free read count. That is, in a given sample, the error rate could vary on the basis of the number of reads sequenced, which might be practically more robust than the shadow regression in which one fixed error rate is provided for a given sample. We also defined a sample-level error rate using the median of the error rates obtained using different numbers of error-free read counts and corresponding fitted shadow counts.

Both the proposed empirical approach and the shadow regression can be affected by outliers. Therefore, we suggest pre-processing the sequence data before data analysis using standard statistical approaches, such as boxplot rule (i.e., based on the upper and lower quartiles of the data sample distribution), chi-squared test [43], Dixon test [44], and Grubbs' test [45]. One can also consider alternative approaches that might be more robust to outliers such as the quantile regression [46] or Akima spline $[47,48]$. In this study, we showed the application of the proposed approaches to data from several sequencing experiments, such as DNA sequencing, mRNA sequencing and re-sequencing. We also showed that the shadow-based approaches are valid when polymorphisms and duplications are present. Because the shadow-based approaches (i.e., shadow regression and our proposed approaches) are independent of the reference genome, they can be applied to other types of sequencing experiments, such as extensive polymorphisms, isoforms, or the microbiome. In particular, as the approaches consider only the reads that differ from the error-free reads by up to two bases as the shadows (i.e., reads with errors), the reads with many differences, such as extensive polymorphisms, isoforms or microbiome data, will not be counted as shadows in the analysis.

\section{Conclusion}

In summary, we proposed an empirical error rate estimation approach in which cubic and robust smoothing splines were used to model the read-shadow relationship. The proposed approach does not assume a linear relationship between the error-free reads and shadows counts and provides more accurate estimations of error rates for next-generation, short-read sequencing data.

\section{Availability of data and materials}

The datasets of MAQC, re-sequencing and ENCODE used for the analyses described in this paper were obtained from National Center for Biotechnology Information Sequence Read Archive (Accession numbers: SRX016366 and SRX016368 for MAQC; SRX000570 for ENCODE; and SRX012886, SRX012887, SRX012888, SRX012889, SRX012890, SRX012891, SRX012892, SRX 012893, SRX012894, SRX012895, SRX012896, SRX012 897, SRX012898, SRX012899, SRX012900, SRX012901, SRX012902, SRX012903, SRX012904, SRX012905, SRX 012906, SRX012907, SRX012908, and SRX012909 for mutation screening). The bacteriophage PhiX 174RF1 (PhiX) DNA data were generated by the Center for Cancer Computational Biology at Dana-Farber Cancer Institute and provided by Drs. Xin Victoria Wang and Giovanni Parmigiani, Dana-Farber Cancer Institute. The data supporting the results of this article are included within the article and its additional files: Additional files $1,2,3$ and 4 which are referenced in the main text.

\section{Additional files}

Additional file 1: Sample sequencing data from MAQC, mutation screening re-sequencing, ENCODE, and PhiX DNA data sets. (DOCX $562 \mathrm{~kb}$ )

Additional file 2: Read counts for the SRR032577 sample in the mutation screening re-sequencing study. (DOCX $17 \mathrm{~kb}$ )

Additional file 3: Sample SRR037440 from the MAQC brain experiment 2 data set and corresponding simulated data using frequency-based and Wang et al. simulation approaches. (DOCX 879 kb)

Additional file 4: Comparisons of different simulation approaches. (DOCX $20 \mathrm{~kb}$ )

\section{Competing interests \\ The authors declare that they have no competing interests.}

\section{Authors' contributions}

XZ co-developed the statistical approach, performed the simulation studies, and drafted the manuscript. JW co-developed the statistical approach, performed the simulation studies, and drafted the manuscript. BP simulated the sequencing data, provided advice on the simulations, and wrote part of the manuscript. SS conceptualized the study, provided advice on the method development, helped to draft the manuscript, and provided critical revisions for important intellectual content. All authors read and approved the final manuscript.

\section{Acknowledgements}

This work was supported in part by National Institutes of Health [NIH grant number 1R01CA131324 (S. Shete), R01DE022891 (S. Shete), R25DA026120 (S. Shete), R03 CA192197 (J. Wang)], and Cancer Prevention Research 
Institute of Texas grant RP130123 (S. Shete)]. This research was supported, in part, by Barnhart Family Distinguished Professorship in Targeted Therapy (S. Shete) and by the National Institutes of Health through Cancer Center Support Grant P30CA016672. The datasets used for the analyses described in this paper were obtained from National Center for Biotechnology Information Sequence Read Archive (Accession numbers: SRX016366 and SRX016368 for MAQC; SRX000570 for ENCODE; and SRX012886, SRX012887, SRX012888, SRX012889, SRX012890, SRX012891, SRX012892, SRX012893, SRX012894, SRX012895, SRX012896, SRX012897, SRX012898, SRX012899, SRX012900, SRX012901, SRX012902, SRX012903, SRX012904, SRX012905, SRX012906, SRX012907, SRX012908, and SRX012909 for mutation screening). We thank Drs. Xin Victoria Wang and Giovanni Parmigiani for their comments on an earlier version of the manuscript and sharing the data from bacteriophage PhiX DNA samples.

\section{Author details}

'Department of Biostatistics, The University of Texas MD Anderson Cancer Center, Houston, TX 77030, USA. ${ }^{2}$ Department of Bioinformatics \& Computational Biology, The University of Texas MD Anderson Cancer Center, Houston, TX 77030, USA. ${ }^{3}$ Department of Epidemiology, The University of Texas MD Anderson Cancer Center, Houston, TX 77030, USA.

\section{Received: 18 August 2015 Accepted: 14 April 2016}

\section{Published online: 22 April 2016}

\section{References}

1. Next-generation sequencing. http://www.nature.com/subjects/nextgeneration-sequencing. Accessed Feb 2015.

2. Turnbaugh PJ, Hamady M, Yatsunenko T, Cantarel BL, Duncan A, Ley RE, et al. A core gut microbiome in obese and lean twins. Nature. 2009; 457(7228):480-4.

3. van Dijk EL, Auger H, Jaszczyszyn $Y$, Thermes $C$. Ten years of next-generation sequencing technology. Trends Genet. 2014;30(9):418-26.

4. Schlotterer C, Tobler R, Kofler R, Nolte V. Sequencing pools of individuals - mining genome-wide polymorphism data without big funding. Nat Rev Genet. 2014;15(11):749-63.

5. Mardis ER. Next-generation sequencing platforms. Annu Rev Anal Chem. 2013;6:287-303

6. Yang Y, Xie B, Yan J. Application of next-generation sequencing technology in forensic science. Genomics Proteomics Bioinformatics. 2014;12(5):190-7.

7. Wang XV, Blades N, Ding J, Sultana R, Parmigiani G. Estimation of sequencing error rates in short reads. BMC Bioinformatics. 2012;13:185.

8. Shendure J, Ji H. Next-generation DNA sequencing. Nat Biotechnol. 2008; 26(10):1135-45.

9. Metzker ML. Sequencing technologies - the next generation. Nat Rev Genet. 2010;11(1):31-46.

10. Jeck WR, Reinhardt JA, Baltrus DA, Hickenbotham MT, Magrini V, Mardis ER, et al. Extending assembly of short DNA sequences to handle error. Bioinformatics. 2007;23(21):2942-4.

11. Sundquist A, Ronaghi M, Tang HX, Pevzner P, Batzoglou S. Whole-genome sequencing and assembly with high-throughput, short-read technologies. PLOS ONE. 2007;2(5):e484.

12. Hert DG, Fredlake CP, Barron AE. Advantages and limitations of next-generation sequencing technologies: a comparison of electrophoresis and non-electrophoresis methods. Electrophoresis. 2008;29(23):4618-26.

13. Brown T, Howe A, Zhang Q, Pyrkosz AB, Brom TH. A reference-free algorithm for computational normalization of shotgun sequencing data. arXiv:1203.4802 [q-bio.GN]. 2012.

14. Hoffmann S, Otto C, Kurtz S, Sharma CM, Khaitovich P, Vogel J, et al. Fast mapping of short sequences with mismatches, insertions and deletions using index structures. PLoS Comput Biol. 2009;5(9):e1000502.

15. Simpson JT. Exploring genome characteristics and sequence quality without a reference. Bioinformatics. 2014;30(9):1228-35.

16. FastQC. A quality control tool for high throughput sequence data. http:// www.bioinformatics.babraham.ac.uk/projects/fastqc. Accessed Feb 2015.

17. Trivedi UH, Cezard T, Bridgett S, Montazam A, Nichols J, Blaxter M, et al. Quality control of next-generation sequencing data without a reference. Front Genet. 2014;5:111.

18. Bullard JH, Purdom E, Hansen KD, Dudoit S. Evaluation of statistical methods for normalization and differential expression in mRNA-Seq experiments. BMC Bioinformatics. 2010;11:94.
19. Butler J, MacCallum I, Kleber M, Shlyakhter IA, Belmonte MK, Lander ES, et al ALLPATHS: de novo assembly of whole-genome shotgun microreads. Genome Res. 2008;18(5):810-20.

20. Schroder J, Schroder H, Puglisi SJ, Sinha R, Schmidt B. SHREC: a short-read error correction method. Bioinformatics. 2009;25(17):2157-63.

21. Kelley DR, Schatz MC, Salzberg SL. Quake: quality-aware detection and correction of sequencing errors. Genome Biol. 2010;11(11):R116.

22. Salmela L. Correction of sequencing errors in a mixed set of reads. Bioinformatics. 2010;26(10):1284-90.

23. Schroder J, Bailey J, Conway T, Zobel J. Reference-free validation of short read data. PLoS One. 2010;5(9):e12681.

24. Li R, Zhu H, Ruan J, Qian W, Fang X, Shi Z, et al. De novo assembly of human genomes with massively parallel short read sequencing. Genome Res. 2010;20(2):265-72

25. Keele L. Semiparametric Regression for the Social Sciences. Chichester, England: John Wiley \& Sons Ltd; 2008.

26. Schröder J, Bailey J, Conway T, Zobel J. Reference-free validation of short read data. PLoS ONE. 2010;5(9):e12681.

27. Gunewardena SS. Optimum-time, optimum-space, algorithms for k-mer analysis of whole genome sequences. J Bioinfo Comp Genom. 2014;1:1-12.

28. Melsted P, Pritchard JK. Efficient counting of $k$-mers in DNA sequences using a bloom filter. BMC Bioinformatics. 2011;12:333.

29. Heo Y, Wu XL, Chen D, Ma J, Hwu WM. BLESS: bloom filter-based error correction solution for high-throughput sequencing reads. Bioinformatics. 2014;30(10):1354-62.

30. Shi L, Reid LH, Jones WD, Shippy R, Warrington JA, Baker SC, et al. The MicroArray Quality Control (MAQC) project shows inter- and intraplatform reproducibility of gene expression measurements. Nat Biotechnol. 2006; 24(9):1151-61.

31. Hu H, Wrogemann K, Kalscheuer V, Tzschach A, Richard H, Haas SA, et al. Mutation screening in 86 known $X$-linked mental retardation genes by droplet-based multiplex PCR and massive parallel sequencing. Hugo J. 2009; 3(1-4):41-9.

32. Birney E, Stamatoyannopoulos JA, Dutta A, Guigo R, Gingeras TR, Margulies EH, et al. Identification and analysis of functional elements in $1 \%$ of the human genome by the ENCODE pilot project. Nature. 2007;447(7146):799-816.

33. Fox J. Nonparametric simple regression: smoothing scatterplots. In: Quantitative Applications in the Social Sciences. Thousand Oaks: Sage; 2000.

34. Pollock DSG, Green RC, Nguyen T. Handbook of Time Series Analysis, Signal Processing, and Dynamics (Signal Processing and its Applications). London: Academic Press; 1999

35. Reinsch CH. Smoothing by spline functions. Numer Math. 1967;10:177-183.

36. Pollock DSG. A handbook of time-series analysis, signal processing and dynamics. San Diego: Academic; 1999.

37. H. Bengtsson. aroma - an R object-oriented microarray analysis environment. http://www1.maths.lth.se/help/R/aroma. Accessed Aug 2014.

38. Bengtsson $\mathrm{H}$, Hossjer O. Methodological study of affine transformations of gene expression data with proposed robust non-parametric multidimensional normalization method. BMC Bioinformatics. 2006;7:100.

39. Leinonen $\mathrm{R}$, Sugawara $\mathrm{H}$, Shumway M. International nucleotide sequence database C: the sequence read archive. Nucleic Acids Res. 2011; 39(Database issue):D19-21.

40. McKenna R, Xia D, Willingmann P, llag LL, Krishnaswamy S, Rossmann MG, et al. Atomic structure of single-stranded DNA bacteriophage $\Phi \times 174$ and its functional implications. Nature. 1992;355(6356):137-43.

41. Sanger F, Air G, Barrell B, Brown N, Coulson A, Fiddes C, et al. Nucleotide sequence of bacteriophage phi X174 DNA. Nature. 1977;265:687-95.

42. Shaw DC, Walker JE, Northrop FD, Barrell BG, Godson GN, Fiddes JC. Gene K, a new overlapping gene in bacteriophage G4. Nature. 1978;272(5653):510-15.

43. Dixon WJ. Analysis of extreme values. Ann Math Statist. 1950;21(4):488-506.

44. Dixon WJ. Ratios involving extreme values. Ann Math Statist. 1951;22(1):68-78.

45. Grubbs FE. Sample criteria for testing outlying observations. Ann Math Statist. 1950;21(1):27-58.

46. Wei Y, Pere A, Koenker R, He X. Quantile regression methods for reference growth charts. Stat Med. 2006;25(8):1369-82.

47. Akima $\mathrm{H}$. A new method of interpolation and smooth curve fitting based on local procedures. J Assoc Comput Machin. 1970;17(4):589-602.

48. Knott GD. Interpolating Cubic Splines. New York: Springer-Science + Business Media, LLC; 2000. 